\title{
The True Height of the Waist: Explorations of Automated Body Scanner Waist Definitions of the TC2 Scanner
}

\author{
Simeon GILL ${ }^{\star a}$, Christopher J. PARKER ${ }^{\star a}$, Steve HAYES ${ }^{b}$, Kathryn BROWNBRIDGE ${ }^{\mathrm{b}}$, \\ Paula WREN ${ }^{\mathrm{b}}$, Anastasiia PANCHENKO ${ }^{\mathrm{b}}$ \\ ${ }^{a}$ The University of Manchester, Manchester, UK; \\ ${ }^{\mathrm{b}}$ Manchester Metropolitan University, Manchester, UK \\ http://dx.doi.org/10.15221/14.055
}

\begin{abstract}
The waist is considered as an important measurement point for clothing and represents part of a control section from which lower body garments hang and garments are often required to fit. However, unlike many measurement sites on the body the waist has no single landmark from which to reference it for a population. The non-contact nature of body scanning further complicates this, though automated measurement systems connected to body scanning have developed methods to determine the waist relative to surface geometry. Whilst body scanning provides an opportunity to analyse large volumes of data, there is limited knowledge of how differences in waist definitions using automated systems of measurement compare to waist placement with reference to accepted upper and lower limits. This work employed content analysis methods to understand existing clothing waist definitions and developed different waist definitions within automated measurement software of a TC2 scanner. Measurement extraction using this explorative set of definitions was undertaken on a sample of 106 females whose upper and lower waist limits had been determined through measurement. The data was then analysed to establish how the definitions compared to the waist height determined as the midpoint between the upper (lowest palpable rib) and lower (highest point of the right iliac crest) limits. It was possible to establish that proportional relationships between lengths that could help in defining waist placement and provide checks in automated extraction. As a result of this study a number of suitable waist definitions are proposed for use in the automated measurement software of body scanning technology.
\end{abstract}

Keywords: 3D body scanning, product development, fashion education, anthropometrics.

\section{Introduction}

Methods of defining the waist placement are contested within existing literature and depend on application, historic approaches, and the skills and tools of those recording dimensions. The introduction of body scanning technology offers the opportunity to collect greater depths of data from individuals [1], but brings unique difficulties in defining the waist. This is especially true when using automated measurement extraction facilities, which cannot reference palpable body landmarks, commonly used to define measurement placement in manual methods [2], [3].

This research explores definitions of the waist in the software of the TC2 KX16 body scanner, which uses non-contact methods to define landmark and measurement positions. Existing definitions of the waist within the software as well as programmed exploratory definitions were used to see how these compare to known waist centres based on traditional manual regions defining the waist. 106 body scans were captured, with participants having their upper and lower limits of the waist region recorded manually to enable comparison between scan extracted dimensions and those from the body scanner software. This research focused on defining the waist height and central waist region and testing the accuracy of a commonly used waist definition within the TC2 software [4] - Waist at smallest horizontal circumference $+4 \mathrm{~cm}$ - against the known waist centre and a number of experimental definitions; driven by analysis of existing waist definitions used in product development.

\subsection{Key research questions}

The research questions addressed within this paper are:

1. How does the height of the different waist definitions compare to the waist centre, defined as the midpoint between the upper and lower limits?

2. Are the upper and lower limits of the waist centre region predictable from existing definitions of the waist height?

3. How suitable is the smallest/narrowest part of the torso as a definition for waist placement?

4. Can the waist height be predicted from other body heights, Centre Back (CB) neck/nape, crotch, hip, and height? 


\section{Existing understandings of the waist definition}

The human waist is an important measurement for product development and can be used in communication of sizing for garments fitting the upper and lower body [5]. Waist measurements are also used extensively in the creation of garment patterns [6]-[8]. However, the wide range of waist placements used in different disciplines demonstrates the lack of a wide reaching agreed definition. Additionally, with the introduction of body scanning this is further complicated due to non-contact methods which cannot utilise traditional landmarking methods where palpation is used to locate skeletal or soft tissue points to help place measurements [9]. The TC2 scanner and surveys using non contact scanners and automated measurement often utilise the small of the back when defining the waist [10], this landmark is linked to the spinal curvature, muscle structure and can be recognised in many cases from the surface geometry. The non-contact nature of body scanning presents many challenges for developing measurement practice from manual to body scanning methods. Whilst this paper focuses on product development applications (specifically clothing), measurement definitions are influenced by multiple users and are subject to varied definitions [11]-[14],until there are clear and detailed methods for all applications requiring waist measurements the definition of the waist will remain an area of potential contention.

\subsection{Defining the waist in anthropometrics/ergonomics}

Standards exist defining the waist as a key measurement site on the body. International standards define the waist girth as: 'girth of the natural waistline between the top of the hip bones (iliac crests) and the lower ribs, measured with the subject breathing normally and standing erect with the abdomen relaxed' [3], [15]; or 'Circumference of trunk at a level midway between the lowest ribs and the upper iliac crest.' [2]. Definitions are highlighted in Figure 1. These clearly set an upper and lower limit, though do not offer further specifics on method for determining the central location, the pitch of the waist circumference, if it deviates from horizontal or the path the measurement should take around the body in reference to immediate landmarks.

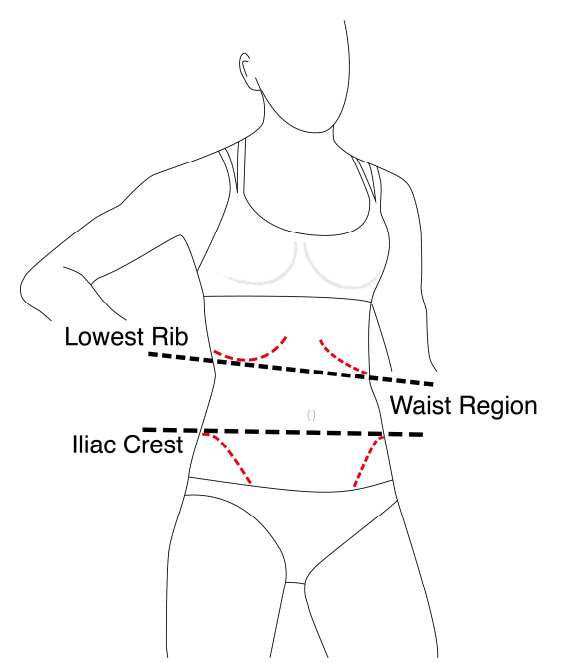

Figure 1. Location of the Human Waist Region ${ }^{1}$

\subsection{Defining the waist for product development and clothing}

Product development methods offer little detail in exact locations of the waist and provide definitions that include terms like natural waistline (Table 2). However the waist remains a vitally important measurement in product development and is used in the majority of methods for creating clothing patterns [6]-[8]. The waist is often viewed as a natural centre of the body that the female figure in classic hourglass descriptions indicates the smallest point in the tapering of the torso between the bust and the hips. This classic interpretation of the waist, strongly influential in the initial steps of product development [16] highlights an area of contention when trying to define the waist on a varied population which contains a number of accepted figure shapes.

\footnotetext{
${ }^{1}$ Illustration based on Hypnotica Studios Infinite [23], Attribution 2.0 Generic https://creativecommons.org/licenses/by/2.0/
} 


\section{Method: Development of tools for assessing the waist region}

To address difficulties in defining the waist location in body scanning this research investigated varied definitions of the waist for clothing product development and those used in the communication of sizing by different retailers. The focus of this work was restricted to women's waists as gaining sufficient levels of male data was difficult.

\subsection{Collection of sample data}

A total of 106 body scans were collected during a 1-year period at a number of scanning events, where the heights of the upper and lower limits of the waist were also manually recorded. The data was collected from a convenience sample of women taking part in scanning events where there was sufficient time to capture their scans as well as to manually record the height of the upper and lower waist region landmarks. At the time of body scan capture; the height and weight were taken for each participant and key characteristics (age, ethnicity) recorded to support later analysis. The body shape of each participant was also determined in line with the methods used in the FFIT system [17].

Body scans were captured using a TC2 body scanner in the regular scan posture (feet approx. $40 \mathrm{~cm}$ apart), whilst manual heights were collected with a harpenden anthropometer connected to a base board with marking to ensure feet were spaced equally to the scan posture. The upper and lower waist landmarks (Table 1) were located on each subject, initially by guided self-location using proprioception, and then checked by the experienced measurement technician using palpation. Definitions for these landmarks are based on the existing measurement standards [2], [3] and are further informed by details of landmark descriptions [18], [19]. Finally the height was recorded with the anthropometer. Landmark heights were recorded to the nearest millimetre with the lower arm of the anthropometer placed at the height of the landmark.

Table 1. Manual definitions for recording the upper and lower waist limits

\begin{tabular}{l|l}
\hline Body Region & Definition \\
\hline $\begin{array}{l}\text { Location of the } \\
\text { lowest rib }\end{array}$ & $\begin{array}{l}\text { Participants first use their fingers to find the lowest lateral palpable rib on their right } \\
\text { side. The measurer then uses this approximate location to start palpation. } \\
\text { Participant places their right hand on their left shoulder, when measurer is satisfied } \\
\text { with LM location then participant places their left finger on the LM and the } \\
\text { measurer records the height using and anthropometer. }\end{array}$ \\
\hline $\begin{array}{l}\text { Location of the } \\
\text { iliac crest }\end{array}$ & $\begin{array}{l}\text { Participants first use their fingers to find the highest lateral point of the iliac crest on } \\
\text { their right side, the measurer then uses this approximate location to start palpation. } \\
\text { Participant places their right hand on their left shoulder, when measurer is satisfied } \\
\text { with LM location then participant places their left finger on the LM and the } \\
\text { measurer records the height using and anthropometer. }\end{array}$ \\
\hline $\begin{array}{l}\text { Posture } \\
\text { Guidance }\end{array}$ & $\begin{array}{l}\text { Participants are requested to remain facing forward and standing erect whilst the } \\
\text { measurements are recorded. }\end{array}$ \\
\hline
\end{tabular}

\subsection{Analysis of waist definitions and development of research tool}

Applying content analysis methods, the different waist definitions from three main sources were analysed; 1) those used on retailer websites, 2) those included in pattern books for product development, and 3) those available in standards and guidance documents related to anthropometrics for product development. Retailer guides on waist definitions were taken from their online pages where guidance is provided to customers on size selection, whilst the other sources were located through analysis of online resources and the holdings of two academic libraries, where product development is taught. The results are presented below in Table 2. 
Table 2. Results of content analysis of waist definitions for product development

\begin{tabular}{ll|ccccccc}
\hline & \multicolumn{2}{|c}{} & \multicolumn{3}{c}{ Measurement } \\
Guides and \\
\end{tabular}

Content analysis enabled seven distinct categories to be created relating to how the waist is defined, with nine percent of sources not providing clear guidance and only sixteen percent recommending the use of body landmarks. The assumption of a natural waist and the waist being relative to the narrowest part of the torso account for at least half of the definitions, though the more developed guidance and standards all clearly related its position to body landmarks. Only a small number (three percent) related waist position to fit preferences; an important consideration as clothing must be worn.

The results of the content analysis (Table 3) suggested the development of sixteen waist definitions within the TC2 software (definitions 2-17), which can be categorised as those relative to the small of back (SOB), those proportionally relative to heights or segment lengths and those related to the shape of the torso. Definition 1 was determined from the recorded heights of the upper and lower waist limits and provided the control from which to assess the other sixteen definitions.

Table 3. Waist Placement Definitions

\begin{tabular}{l|l|l|l}
\hline No. & Waist Placement Definition & No. & Waist Placement Definition \\
\hline 1 & Height of Mid Waist Position (Midway [WR1] and & 10 & Small of the Back +6cm_Height_Back \\
& [WR2] & & \\
2 & Scan Waist Height (Small of the Back $+4 \mathrm{~cm})$ & 11 & Small of the Back +8cm_Height_Back \\
3 & Waist_68\%_to_CB_neck_Height & 12 & Small of the Back +10cm_Height_Back \\
4 & Waist_Start68\%+4cm_CB_neck_Height_Bk & 13 & Small of the Back +12cm_Height_Back \\
5 & Waist_70\%_to_CB_neck_Height & 14 & Small of the Back -2cm_Height_Back \\
6 & Waist_72\%_to_CB_neck_Height & 15 & Small of the Back -4cm_Height_Back \\
7 & Waist_Start72\%+4cm_CB_neck_Height_Bk & 16 & Waist-Narrowest_Height_Back \\
8 & Small of the Back_Height_Back & 17 & Waist_Narrowest-4cm_Height_Back \\
9 & Small of the Back+2cm_Height_Back & & \\
\hline
\end{tabular}

These definitions were created within the TC2 body scanning software and the data batch processed from the 106 scans of female participants. Data was collected in excel and then processed statistically within SPSS [20].

\section{Results and Analysis}

\subsection{Descriptive Statistics}

Within this study, 106 females aged 18-71 (Mean $=28, S D=10.76)$ were scanned, with a wide range of heights $(154-178 \mathrm{~cm}$, Mean $=165, \mathrm{SD}=5.39)$ and weights $(40.1-117.6 \mathrm{Kg}$, Mean $=64.68, \mathrm{SD}=$ 12.96). As demonstrated by Error! Reference source not found., a wide range of body shapes were found in the sample, which was predominantly of healthy to Extremely Slim body-fat levels; see Figure 3. Consequently, the sample can be considered as representative of the UK population. However, the sample contains a lower percentage of obese persons, currently estimated to be $57 \%$ of adult females in the UK [21]. 


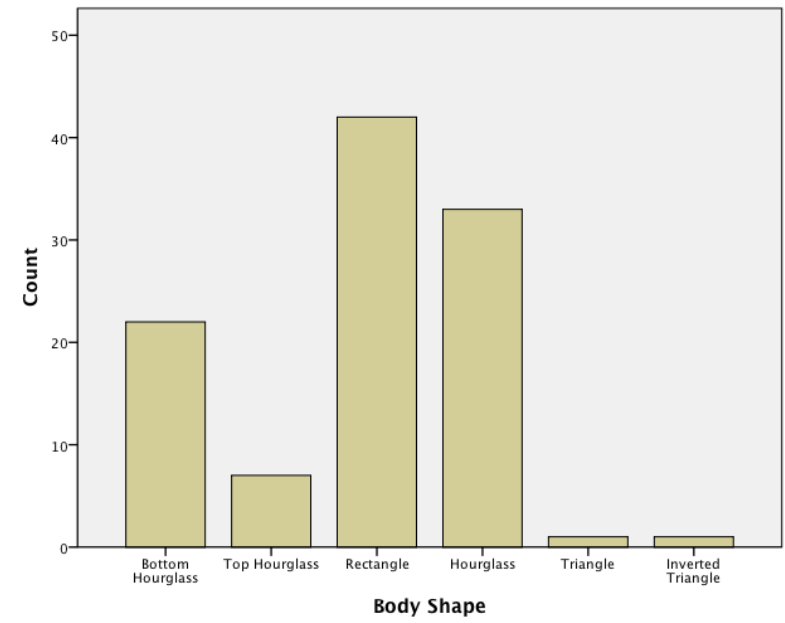

Figure 2. Frequency of Participant Body Shapes

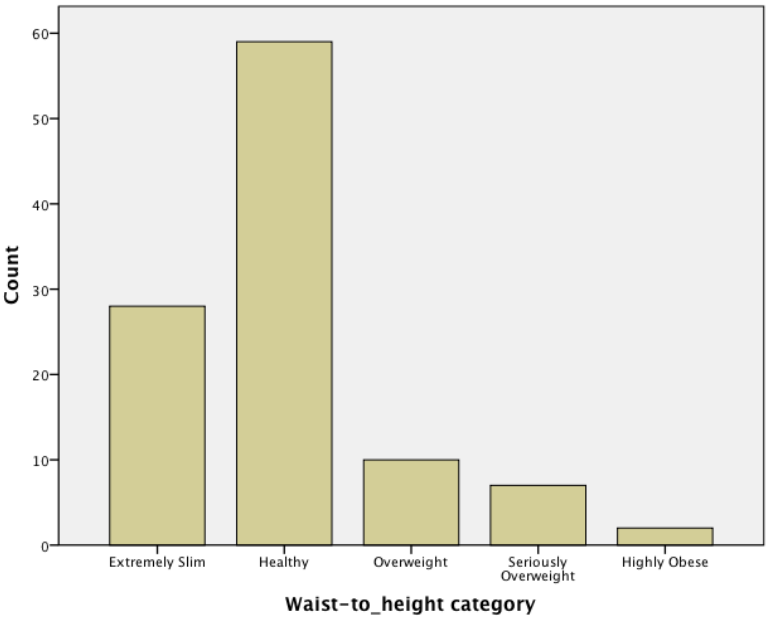

Figure 3. Frequency of Participant Waist-to-height Ratio Categories

\subsubsection{Central Waist Region Length of the Sample}

Considering the range and limitations of the central waist region (see Figure. 1), starting at the iliac crest between 55.49 and $63.77 \%$ of the persons height $(M=59.89, S D=1.49)$, and ends at the lowest palpable rib between 59.58 and $69.01 \%$ of the persons height $(M=64.09, S D=1.41)$. Consequently, the mean length of the region was between 2.4 and $13.4 \mathrm{~cm}(M=6.92, S D=2.23)$.

There is a clear relationship between the limits of the central waist region and the overall height of the participants; however, the physical length of the central waist region can be seen to vary significantly, representing the natural variation within a population. This suggests that predicting the region from height alone may be limited, though there is the possibility of using multiple heights to get a better understanding of where the central waist region may be, there is an evident requirement to determine how the central waist region relates to crotch, CB neck and other key heights which are discernible from the scan outputs and also whether this has a relationship to key classification like age and body shape. An indication of the range of waist regions observed within the data set is presented below is Figures 4 and 5.

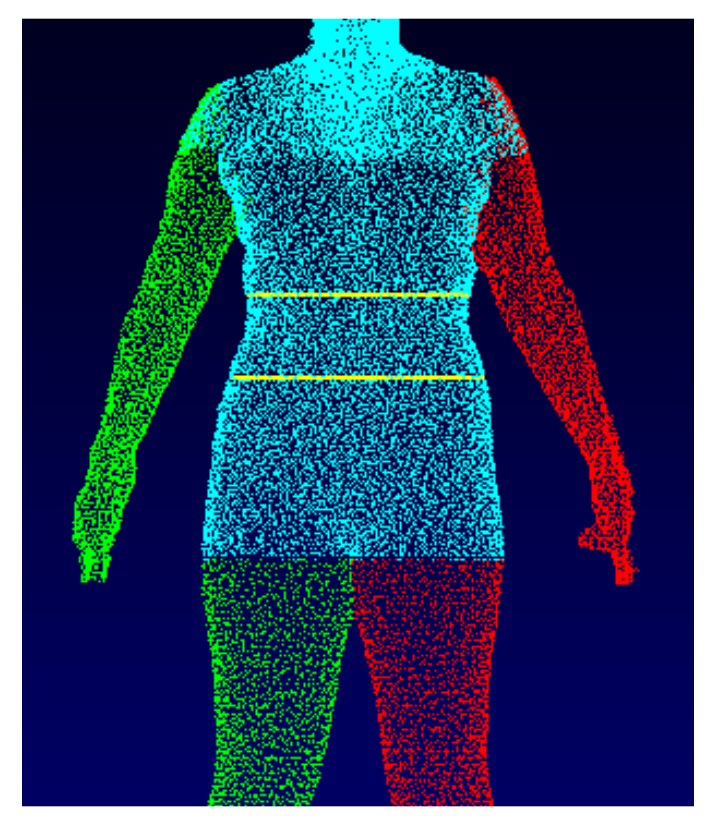

Figure 4. Sample scan with a large central waist region

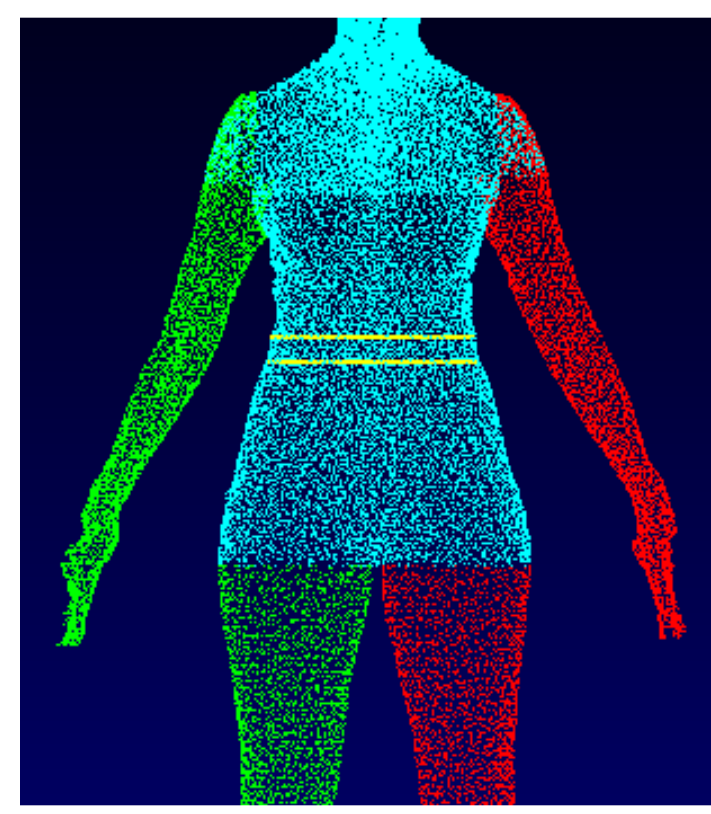

Figure 5. Sample scan with a small central waist region 


\subsubsection{Sample Waist Height}

Within this study, 17 different definitions for the height of the waist were considered, which when expressed as a percentage of total body height, exhibit a range of means from $58.51 \%$ (Waist Height $3, S D=.73$ ) to $64.51 \%$ (Waist Height $17, S D=3.46$ ). The range and frequency of waist heights (as a percentage of the participants' total height) for waist definition 2, commonly used in body scanning is described in Figure 6Figure 6. These indicate a variation as with the central waist region and again further understanding is required to determine what predictors may help narrow the definition of the waist in body scanning.

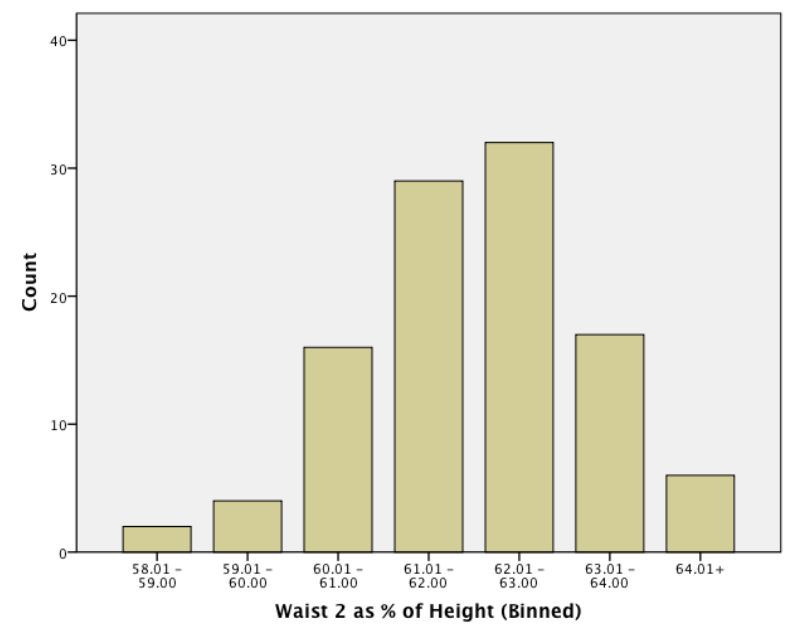

Figure 6. Bar chart demonstrating Waist 2 locations

\subsubsection{Sample Waist Circumferences}

Finally, the differences in waist circumferences need to be considered. The circumferences measured at the 17 locations for the waist produced a range of means from $77.44 \mathrm{~cm}$ (location $13, S D=9.64$ ) to $90.07 \mathrm{~cm}$ (location $3, S D=11.04$ ). This clearly show, as with existing research [14] that the definition for waist location will return significantly different values depending where on the tapering torso it is located. This highlights the difficulties of inappropriate waist placement and the level of error that may occur when trying to apply them in product development practice.

\subsection{How does $\mathrm{SOB}+4 \mathrm{~cm}$ compare to the central waist?}

A paired sample t-test was conducted to evaluate the relationship between the height of the centre of the central waist region and the definition 'waist at $\mathrm{SOB}+4 \mathrm{~cm}$ '. This pairing exhibited statistical significance $(t(105)=-8.16, p=.000)$, demonstrating the significant difference from the centre of the central waist region, with $95 \%$ confidence intervals between 2.17 and $1.32 \mathrm{~cm}$ below the centre point $(M=1.75 \mathrm{~cm}, S D=2.20)$ with a medium effect size (.39). This indicates that the smallest circumference in the central waist region does not always equate to the centre (mid point) of the central waist region and highlights a potential conflict between data required to drive product development and standardisation of waist placement for comparing populations.

\subsection{Placing the Waist Measurements in the centre of the region}

As the most commonly held definition of the waist is between the lowest lateral palpable rib and highest lateral aspect of the iliac crest it is important to establish how waist definitions relate to what may be termed the Central Waist Region (CWR). The following sections explore how the different definitions of the waist and their respective heights compare to the central waist region and allow an understanding of their suitability to define the waist using automated measurement extraction in body scanning.

\subsubsection{Placement of the Central Waist}

A series of paired-sample t-tests were conducted to evaluate the relationship between the centre of the waist (as defined as the central point between the lowest palpable rib and the iliac crest) and waist definitions 2-17 within this study. All pairs (except waist definitions 6 and 9) exhibited statistical significance, demonstrating their significant difference from the central waist definition. Of these, waist definition Nine $(M=103.87, S D=4.49)$ was the most statistically similar to the waist centre definition 
$(M=102.12, S D=4.14), t(105)=.091, p=.928$. The mean difference between the measurements was $.022 \mathrm{~cm}$ with a $95 \%$ confidence interval ranging from -2.17 to -1.32 . The eta squared statistic $(<.000)$ indicated an almost undetectable effect size.

Consequently the waist definition being the statistically closest to the 'centre of the central waist region' measurement is Definition Nine: 'the Small of the Back $+2 \mathrm{~cm}$ '. This is interesting as when calculating the mean distance from the Small of the Back to the central waist height within the sample is $2.02 \mathrm{~cm}(\mathrm{SD}=2.26)$. However, as Figure 7 highlights, the search for a definitive calculation to predict the waist location in the wider population must be considered elusive due to the wide range of waist placements occurring in nature. There is an evident requirement to explore further relationships between multiple dimensions and other classifiers, like height and age to establish how these effect the waist placement within the population.

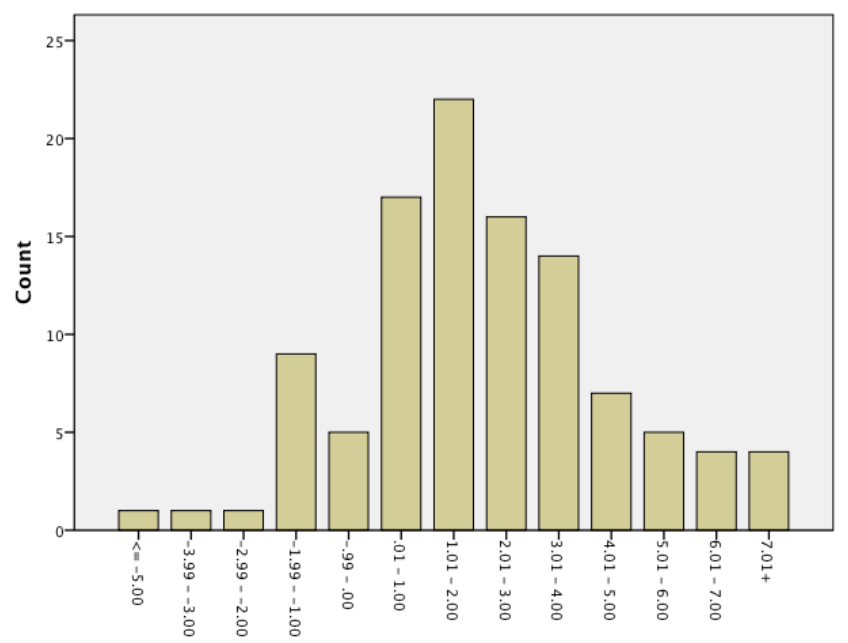

Figure 7. Distances from Small of Back $(\mathrm{SoB})$ to Waist centre in the Sample $(\mathrm{cm})$

\subsubsection{Lowest Palpable Rib}

A series of paired-sample t-tests were conducted to evaluate the relationship between the placement of the lowest palpable rib on the participant and waist definitions 2-17 within this study. Of these, five waist placement definitions Seven $(M=105.22, S D=3.85)$, Twelve $(M=10.60, S D=4.27)$, Thirteen $(M=105.81, S D=4.21)$, Fourteen $(M=105.84, S D=4.20)$ and Seventeen $(M=106.26, S D=6.86)$ did not achieved statistical significance with the placement of the rib $(M=105.58, S D=4.39)$, suggesting degrees of significant similarity in measurement; see Table 4. While Waist Placement definition Eleven was statistically different from the placement of the rib, it is included within this section since its mean scores $(M=105.05, S D=4.43)$ were close enough to the rib placement to be effectively a predictor of its location.

Table 4. Paired Sample t-test demonstrating significant similarity with lowest palpable rib placement

\begin{tabular}{|c|c|c|c|c|c|c|c|c|}
\hline & \multicolumn{8}{|c|}{ Paired Differences } \\
\hline & \multirow[b]{2}{*}{ Mean } & \multirow{2}{*}{$\begin{array}{c}\text { Std. } \\
\text { Deviation }\end{array}$} & \multirow{2}{*}{$\begin{array}{l}\text { Std. Error } \\
\text { Mean }\end{array}$} & \multicolumn{2}{|c|}{$\begin{array}{c}95 \% \text { Confidence Interval of } \\
\text { the Difference }\end{array}$} & \multirow[b]{2}{*}{$t$} & \multirow[b]{2}{*}{ df } & \multirow{2}{*}{$\begin{array}{l}\text { Sig. } \\
\text { (2-tailed) }\end{array}$} \\
\hline & & & & Lower & Upper & & & \\
\hline $\begin{array}{l}\text { Lowest Rib - Waist } \\
\text { Height } 7\end{array}$ & .36 & 1.89 & .18 & -.00 & .72 & 1.97 & 105 & .052 \\
\hline $\begin{array}{l}\text { Lowest Rib - Waist } \\
\text { Height } 11\end{array}$ & .53 & 2.15 & .21 & .11 & .94 & 2.53 & 105 & .013 \\
\hline $\begin{array}{l}\text { Lowest Rib - Waist } \\
\text { Height } 12\end{array}$ & -.02 & 1.88 & .18 & -.38 & .35 & -.09 & 105 & .928 \\
\hline $\begin{array}{l}\text { Lowest Rib - Waist } \\
\text { Height } 13\end{array}$ & -.23 & 1.86 & .18 & -.58 & .13 & -1.24 & 105 & .217 \\
\hline $\begin{array}{l}\text { Lowest Rib - Waist } \\
\text { Height } 14\end{array}$ & -.26 & 1.89 & .18 & -.62 & .10 & -1.41 & 105 & .160 \\
\hline Waist Height 17 & -.68 & 5.44 & .53 & -1.73 & .36 & -1.29 & 105 & .199 \\
\hline
\end{tabular}


Of the waist placement definitions within Table 4, Waist Height Definition 12 can be considered the best predictor of the placement of the lowest palpable rib, with considered statistical significance and a calculated correlation factor of .907. This suggests that the shape of the torso, within at least this sample, tapered to a narrow circumference at a point higher than the central waist location and that there is a more of a relationship between the smallest circumference without suitable controls on its position and the location of the lowest rib. With this in mind it is important to limit the waist placement by other means than an open smallest circumference of the torso. Further to this a method could be investigated for predicting the height of the lowest rib from the scan using automated extraction and provide further methods to locate the waist height in a manner more transparent than current software algorithms and provide further controls to the user. Waist Definition 17 (however) can be considered the worst definition of the waist due to its consistent placement of the waist above the lowest palpable rib by the greatest amount; outside of the waist region. The narrowest point on the torso, which may be visually associated as the waist will often be in the upper part, or in some cases above the central waist region. This would suggest that basing waist placement related to the centre of the central waist region will not be well served in relation to the narrowest part of the torso defined during body scanning.

\subsubsection{Iliac Crest}

A series of paired-sample t-tests were conducted to evaluate the relationship between the placement of the iliac crest on the participant and waist definitions 2-17 within this study. All of the seventeen definitions achieved statistical significance (difference) with the height measurement or the iliac crest. However, when considering the descriptive statistics, Waist Definition Three $(M=96.38, S D=3.22)$ gives a mean location of the waist $2.28 \mathrm{~cm}$ below that of the mean location of the iliac crest $(M=$ 98.66, $S D=4.18)$. While the paired-sample t-test demonstrated that Waist Definition Five $(M=99.22$, $S D=3.54$ ) was statistically significant (different to) with the placement of the iliac crest, $t(105)=-$ $2.59, p<.011$. The mean decrease was -.56 with a $95 \%$ confidence interval ranging from -.99 to 1.05. The eta squared statistic (.06) indicated a negligible effect size.

Consequently, while not a strong predictor, Waist Definition Five can be taken as the strongest locator for the iliac crest of the definitions examined within this study. It is therefore possible to use waist location Five (70\% of the CB neck height) to predict the location of the iliac crest. This can again be used to narrow waist location in a manner accessible to the software user and can be contextualised into existing product development practice.

\subsection{Alternative Measures as Predictors for Waist Height}

Considering the correlation between Central Waist Height and other body measurements, Table 5 demonstrates the series of strong correlations between this central measurement and alternative measurements of the body; e.g. Neck Height. Importantly, this is considering the Central Waist Height as a percentage expression of the alternative measurement. Within these correlations, the Neck Height, Hip A Measurement and Crotch Height are all highly appropriate predictors of Central Waist Height.

Table 5. Correlations between Height of Waist and Body Measurements

\begin{tabular}{ll|lllll}
\hline & & Neck Height & Bust Height & Hip A Height & Hip B Height & Crotch Height \\
\hline Height of & Pearson Correlation & $.895^{\prime \prime}$ & $.833^{\prime \prime}$ & $.893^{\prime \prime}$ & $.673^{\prime \prime}$ & $.899^{\prime \prime}$ \\
waist centre & Sig. (2-tailed) & .000 & .000 & .000 & .000 & .000 \\
& $N$ & 106 & 106 & 106 & 106 & 106 \\
\hline
\end{tabular}

\section{Discussion}

Within this section, the four research questions of this paper are addressed. Although factors such as the participants' fitness, age and ethnicity were considered in the analysis, their non-statistically significant influence means they are not discussed within this section. For further consideration, see Section 6.1.

\subsection{Research Aim 1: Comparison of Waist Height to Waist Centre Definitions}

As shown in Section 4.2, the widely utilised definition of the waist as the 'Small of the Back $+4 \mathrm{~cm}$ is statistically different to the centre of the central waist region; defined as the central point between the 
iliac crest and the lowest palpable rib. Further to this, Section 4.3.1 demonstrated that Waist Definition Nine (Small of the Back $+2 \mathrm{~cm}$ ) bore the closest similarity to the actual height of the waist defined by existing product development standards [2], [3]. This research indicates there is a strong relationship between the small of the back landmark and the waist defined as the centre of the central waist region. However, there is still variation between these heights, which requires further analysis, and consideration of which predictors are required to understand when further steps are required to more accurately define the waist position on participants.

Considering the waist definitions and their ability to predict key locations of the waist, including the skeletally defined waist centre it can be seen there are some good opportunities to use certain definitions to help in classifying the placement of the waist (Error! Reference source not found.). This has important considerations when looking to integrate scanning systems into existing product development practice and the documented methods for defining the waist location that exist and are applied by practitioners. Being able to define the centre of the central waist region could provide a benchmark that other waist definitions could reference in terms of height location, in much the same way a dropped waist may be $4 \mathrm{~cm}$ lower than a natural waist when creating a trouser pattern [6]. Having this central waist location, which could be the small of the back if further evidence exists of its stability within a wider population, would enable users to define different waists in accordance with their practice, but locate these with reference to this point. This would require some further consideration of defining the waist in terms of nomenclature and suggests a means to differentiate the waist and its different definitions, possibly in relation to a stable and consistent waist centre.

\subsection{Research Aim 2: Predictability of Upper and Lower Waist Regions}

Section 4.3.2 demonstrates how while Waist Definition Five is partially similar to the measured Lower lliac Crest landmark height, none of the definitions investigated through this study could be said to be a strong predictor of its location. However, as show in Section 4.3.3, the participants' Lowest Palpable Rib can be derived automatically through the use of Waist Definition 12 (Small of the Back $+10 \mathrm{~cm}$ ). This suggests that despite variation within the population, it is possible to derive such measurements automatically through body scanning, although this has not yet been fully achieved. This also indicates that the smallest circumference may accord with a point higher on the body than the waist and suggests a need for greater anatomical understanding when defining measurement location for product development. This also has an impact on existing industry definitions of the waist, with $44 \%$ suggesting it is at the narrowest point of the torso (Table 2). It is also important that some consideration is given to how data of the waist is applied in clothing product development and whether knowing this smaller waist would be of benefit to pattern construction practice.

\subsection{Research Aim 3: Narrowest Torso Point Compared to Midpoint and Centre of Waist}

The analysis also highlights that a number of waist definitions will not accurately place the waist at the height of the centre of the central waist region. Waist definition Seven, which relates to the narrowest point on the torso when viewed visually front on, defines a waist in the upper part of the central waist region and in certain cases defines a waist above the central waist region. This would mean a waist defined upon the lower parts of the ribcage, which will in many cases be unsuitable for clothing that must remain functional. The anatomy of the body, the deposition of fat and muscle as well as the clear links between the skeletal system and body surface need to be further recognised and referenced when defining measurement locations [22]. Using this data in constructing patterns and body worn products may create difficulties in balance and fit and questions some of the existing theory on body shape, which often references waist location to the narrowest indentation.

\subsection{Research Aim 4: Prediction of Waist Height from other Body Heights}

As shown in Section 4.4, there is strong correlation between the heights of various alternative body measurements (most notably neck and crotch height) that can allow for accurate prediction of the height of the centre of the central waist region. This suggests that body scanning can use multiple locations when defining the waist, which may help narrow the area of focus in a manner understandable by current product development practitioners.

The importance of this outcome is tied closely to that of Research Aims 1 and 2, in that this paper has demonstrated a method of automatically extracting points through inference that are otherwise not inferable through body scanning. Therefore, if Aims 1 and 2 are demonstrated as important to garment design and ergonomics in body scanning, then Aim 4 can be considered as similarly important. However, what is not yet known is which method of measurement inference is most 
suitable, and in which situation. Further to this, these alternative measurements of the body may be used within the software to check the waist height and could be used to flag potential placement errors that may require user intervention. Current scanning systems have few in built checks to help spot errors in landmark location of measurement length, which would typically be checked in manual surveys at the time of data collection.

\section{Conclusion}

From the data analysis within this study, it is clear there is variation in returned values of the waist height, but some opportunities exist to provide greater analytical control to the user in a manner appropriate to existing product development skillsets. There is a central waist region definable by physical landmarks that can be predicted with some level of success here using automated scan methods. There is an absolute waist in the centre of the central waist region that should be maintained as a benchmark and can be used as a relative point when defining different waist levels for product development or variation in accepted practice. Whilst other waist definitions are accepted to exist, they must always be relative to this, or a similar waist benchmark that can provide greater stability when referencing measurement data in isolation from the methods of data collection. There are clear indications that proportional lengths can define the lower limits of the central waist region with reference to landmarks dependent on skeletal structure and proportion. Further to this the narrowest point on the torso defined visually front on is likely to be misleading as to actual waist height and is more likely to be in the upper part of the central waist region or above this. The narrowest point is most likely caused by the rib cage tapering the torso and is not a good predictor of waist height and suggests a need for greater anatomical reasoning when assessing waist position and its height, as well as calling into question some clothing industry practice for defining the waist.

\subsection{Research Limitations}

Due to sample size restrictions, there were a number of limitations within this study. Firstly, since only females were samples, it is not yet known the extent to which the definitions highlighted as having strong predictive powers for points on the human body (Table 5) are replicated in males. Additionally, the relatively low sample size of overweight and obese individuals may have forced a Type I error in the statistics, meaning that no significant influence of body mass was observed, while in fact an influence may exist in the general population (Figure 3). Similarly, not all body shapes were fully represented within the sample size, which may or may not have contributed to a Type I error in this factor (Figure 2). Consequently, practitioners need to be aware of these limitations when applying the findings of this research, and further investigation is needed to fully understand this area.

\subsection{Further Research}

This research has enabled for some clear understanding of the waist and its definition in product development using body scanning. However, there are some further areas that need to be addressed to fully understand this. It is important to determine how the different waist heights affect the waist circumference and the potential impact of an incorrect circumference (too big or too small) on existing methods of product development. Additionally, there is a need for more statistically derived definitions of the human body to determine regions using automated scanning systems that are not easily identifiable as they are traditionally reliant on manual methods involving palpation.

\section{References}

[1] E. Bye, K. L. LaBat, and M. R. Delong, "Analysis of Body Measurement Systems for Apparel", in Clothing and Textiles Research Journal, vol. 24, no. 2, 2006, pp.66-79, DOI: $10.1177 / 0887302 X 0602400202$.

[2] ISO, "7250-1:2010: Basic human body measurements for technological design - Part 1: Body measurement definitions and landmarks", International Standards Organisation, 2010.

[3] ISO, "8559:1989: Garment construction and anthropometric surveys - Body dimensions". International Standards Organisation, 1989.

[4] TC2, "[TC] 2", vol. 7.2. TC2, Cary, NC, USA, 2011.

[5] BSI, "BS EN 13402-2:2002, Size Designation of Clothes - Part 2: Primary and secondary dimensions", British Standards Institute, London, UK, 2002. 
[6] W. Aldrich, Metric pattern cutting for Women's wear, 5th ed. Oxford, UK: Blackwell Publishing, 2008.

[7] H. J. Armstrong, Pattern Making for Fashion Design, 5th ed. Upper Saddle River, N.J.: Pearson/Prentice Hall, 2010.

[8] A. Beazley and T. Bond, Computer-aided pattern design and product development. Oxford, UK: Blackwell Publishing, 2003.

[9] A. Beazley, "Size and fit: Procedures in undertaking a survey of body measurements - Part 1", in Journal of Fashion Marketing and Management, vol. 2, no. 1, 1997, pp.55-85.

[10] E. Kirchdoerfer et al., "Proposed human body measurements standard ", 2002. [Online] www.cs.ucl.ac.uk/staff/p.treleaven/BodyMeasurements.pdf, Accessed 6th Feb 2012

[11] Scanner Waist, bodyscanning, http://bodyscanning.wordpress.com/scanner-waist/, accessed 2014. .

[12] J. Bigaard et al., "Self-Reported and Technician-Measured Waist Circumferences Differ in Middle-Aged Men and Women", in The Journal of Nutrition, vol. 135, no. 9, 2005, pp.22632270.

[13] N. Daniell, T. Olds, and G. Tomkinson, "The importance of site location for girth measurements", in Journal of Sports Sciences, vol. 28, no. 7, 2010, pp.751-757, DOI: 10.1080/02640411003645703.

[14] J. Wang et al., "Comparisons of waist circumferences measured at 4 sites", in American Journal of Clinical Nutrition, vol. 77, no. 2, 2003, pp.379-384.

[15] BSI, "BS EN 13402-1:2001, Size Designation of Clothes - Part 1: Terms, definitions and body measurement procedure", British Standards Institute, London, UK, 2001.

[16] R. Sanderson and S. Gill, "Turning Figure Drawing on its Head", in The power of fashion: 16th annual IFFTI conference, 2014.

[17] J. Y. Lee et al., "Comparison of body shape between USA and Korean women", in International Journal of Clothing Science and Technology, vol. 19, no. 5, 2007, pp.374-391.

[18] D. Field, Anatomy : palpation and surface markings, vol. 3rd. Oxford: Butterworth-Heinemann, 2001.

[19] J. V Basmajian, Surface anatomy : an instruction manual. Baltimore ; London: Williams \& Wilkins, 1983.

[20] IBM, "SPSS", vol. 20. IBM Ltd., USA, 2013.

[21] M. Ng et al., "Global, regional, and national prevalence of overweight and obesity in children and adults during 1980-2013: a systematic analysis for the Global Burden of Disease Study 2013", in Lancet, vol. 384, no. 9945, 2014, pp.766-781, DOI: 10.1016/S0140-6736(14)604608.

[22] S. Gill, "Determination of Functional Ease Allowances using anthropometric measurement for application in pattern construction", Unpublished PhD Thesis, Manchester Metropolitan University, 2009.

[23] All Women Lifeguard Tournament 2013, Flickr, https://www.flickr.com/photos/15119648@N04/9425789904/, accessed 2014. . 